\title{
Aarnoo Análise do processo de eliminação da transmissão da malária na Amazônia brasileira com abordagem espacial da variação da incidência da doença em 2016*
}

doi: $10.5123 /$ S1679-49742018000300010

\author{
Analysis of the process of malaria transmission elimination with a spatial approach to \\ incidence variation in the Brazilian Amazon, 2016
}

\section{Análisis del proceso de eliminación de la transmisión de malaria en la Amazonía brasileña con enfoque espacial en la variación de la incidencia de la enfermedad en 2016}

\author{
Rui Moreira Braz ${ }^{1}$ - (D) orcid.org/0000-0002-3834-578X \\ Christovam Barcellos ${ }^{2}$ \\ ${ }^{1}$ Ministério da Saúde, Secretaria de Vigilância em Saúde, Brasília, DF, Brasil \\ ${ }^{2}$ Fundação Instituto Oswaldo Cruz, Instituto de Comunicação e Informação Científica e Tecnológica em Saúde, Rio de Janeiro, RJ, Brasil
}

\section{Resumo}

Objetivo: identificar áreas com eliminação da transmissão da malária e os níveis de variação da incidência da doença na Amazônia brasileira em 2016, e apresentar indicador de prioridades das ações de controle. Métodos: foi realizado estudo ecológico com dados do Sistema de Informação de Vigilância Epidemiológica da Malária (Sivep-Malária); os municípios foram classificados em três grupos - eliminação alcançada; em processo de eliminação; em busca da redução -; foi criado indicador composto para estratificar os municípios prioritários para ações de controle. Resultados: detectaram-se 337 (41,7\%) municípios com eliminação alcançada, 398 (49,3\%) em processo de eliminação e 73 (9,0\%) em busca da redução; o indicador de prioridades criado identificou 71 municípios, responsáveis por concentrar $95 \%$ dos casos. Conclusão: a grande maioria dos municípios já alcançaram a eliminação da transmissão da malária ou estão em processo de eliminação dessa transmissão; 0 indicador de prioridades poderá contribuir no direcionamento das ações de controle da infecção.

Palavras-chave: Malária; Monitoramento; Análise Espacial; Estudos Ecológicos.

\footnotetext{
*Artigo derivado de trabalho de conclusão de estágio pós-doutoral, intitulado 'Implantação e avaliação do Sistema de Monitoramento da Incidência da Malária (SIMAM) na Região Amazônica: um olhar sobre as populações vulneráveis', apresentado por Rui Moreira Braz junto ao Programa de Pós-Graduação em Informação e Comunicação em Saúde, do Curso de PósDoutorado 'Brasil sem Miséria' da Fundação Instituto Oswaldo Cruz, em 2017.
}

Endereço para correspondência:

Rui Moreira Braz - Ministério da Saúde, Secretaria de Vigilância em Saúde, Departamento de Vigilância das Doenças

Transmissíveis, SRTV 702, Via W5 Norte, Edifício PO700, 6ªndar, Brasília, DF, Brasil. CEP: 70723-040

E-mail:rui.mbraz@gmail.com 


\section{Introdução}

A malária permanece como grande problema de Saúde Pública no Brasil. Responsável por internações, absenteísmos no trabalho e nas escolas, e óbitos, a infecção prejudica o desenvolvimento socioeconômico nas áreas endêmicas. Segundo a Organização Mundial da Saúde (OMS), no ano de 2015, o Brasil registrou o maior número de casos da doença entre todos os países das Américas. ${ }^{1}$

A incidência da malária é influenciada por fatores ambientais (vegetação, clima e hidrologia), sociodemográficos (migrações, densidade populacional, grau de educação formal, cultura, renda e atividade laboral da população), biológicos (espécie e densidade de mosquitos do gêneroAnopheles, espécie de Plasmodium e grau de imunidade da população) e político-organizacionais (divisão territorial, organização e efetividade dos serviços de saúde, ocupação do solo, empreendimentos agropecuários e de infraestrutura). ${ }^{2}$

\section{Análises de informações rotineiras poderão auxiliar os gestores na identificação oportuna das epidemias e de outros indicadores, contribuindo para a redução efetiva da doença nos municípios da região da Amazônia brasileira.}

Os esforços para reduzir a incidência da malária no país remontam a 1898, com Adolfo Lutz, e a 1905, quando Carlos Chagas comprovou a transmissão intradomiciliar da doença. Na década de 1930, o Serviço de Malária do Nordeste contribuiu para a eliminação da epidemia nos estados do Ceará e Rio Grande do Norte. ${ }^{3}$ Nos anos de 1940, estimou-se a ocorrência de 5 milhões de casos de malária. Com a Campanha de Erradicação da Malária iniciada em 1965, sua incidência foi reduzida para 52 mil casos em $1970 .{ }^{4}$ Desde então, houve incrementos sucessivos dos casos, devidos aos projetos de ocupação da Amazônia brasileira que não levaram em conta a estrutura de saúde necessária para assistir as pessoas com malária, culminando com 635 mil casos na região em 1999.5

Vários esforços objetivaram conter a incidência da doença, como a Operação Impacto, em 1986, ${ }^{6}$ o Plano de Intensificação das Ações de Controle da Malária, em $1999,{ }^{7}$ as novas diretrizes do Programa Nacional de Prevenção e Controle da Malária (PNCM), em 2003, ${ }^{5}$ e a parceria com o Fundo Global de luta contra aids, tuberculose e malária, em 2010. ${ }^{8} \mathrm{Em} 2016$, o número de casos novos reduziu-se para 143 mil. A descentralização do controle da malária para estados e municípios, a partir de 2000, também foi importante para a diminuição da transmissão. ${ }^{9}$ Apesar dos avanços, a incidência da malária permanece alta, com mais de 99\% dos casos concentrados na Amazônia brasileira. Encontra-se em execução o Plano de Eliminação da Malária (PEM), ${ }^{10}$ com propostas para erradicação da doença no curto, médio e longo prazos. Contudo, a utilização de critérios de priorização das ações com base em um indicador composto, que considere 0 porte populacional, o número de casos e a incidência parasitária da doença no município, além de outros aspectos como agrupamentos de municípios epidêmicos e duração das epidemias, poderá contribuir para 0 aprimoramento do PEM.

O PNCM conta com o Sistema de Informação de Vigilância Epidemiológica da Malária (Sivep-Malária) ${ }^{11}$ como principal ferramenta de apoio à vigilância e controle da doença. Todavia, são necessários dispositivos para o monitoramento estatístico da malária em localidades vulneráveis, incluindo a identificação do padrão espacial da incidência nessas áreas. Pode-se atribuir a agregação espacial de doença a fatores demográficos, ambientais e/ou socioculturais superpostos geograficamente, quando há uma inesperada aglomeração de casos no espaço e/ou tempo. ${ }^{12}$ Portanto, o conhecimento da estrutura e da dinâmica espacial é importante para a caracterização da situação de saúde da região, ${ }^{13}$ melhor planejamento e alocação dos recursos disponíveis. Análises de informações rotineiras poderão auxiliar os gestores na identificação oportuna das epidemias e de outros indicadores, contribuindo para a redução efetiva da doença nos municípios da região da Amazônia brasileira.

0 presente estudo teve por objetivo identificar áreas com eliminação da transmissão da malária e os níveis de variação da incidência da doença na Amazônia brasileira em 2016, e apresentar um indicador de prioridades para as ações de controle da infecção.

\section{Métodos}

Realizou-se um estudo ecológico com dados do Sivep-Malária referentes aos municípios que compõem a região da Amazônia brasileira. 
A região concentra $99 \%$ dos casos de malária e abrange os estados do Acre (AC), Amapá (AP), Amazonas (AM), Mato Grosso (MT), Pará (PA), Rondônia (R0), Roraima (RR), Tocantins (TO) e parte ocidental do Maranhão (MA) ${ }^{14}$ Estes nove estados possuem 808 municípios incluídos na área de estudo; desses municípios, 52 fazem fronteira com a Bolívia, Colômbia, Guiana, Guiana Francesa, Peru, Suriname e Venezuela. A Amazônia abrange 59\% do território brasileiro e somava, de acordo com o Censo Demográfico da Fundação Instituto Brasileiro de Geografia e Estatística (IBGE), 25.469.352 hab. em 2010.

Para identificação dos níveis de variação da incidência da malária, foi utilizado o diagrama de controle por quartis, com dados dos sete anos anteriores ao período de monitoramento de 2016, adaptado a partir de estudo realizado no município de Cruzeiro do Sul (AC). ${ }^{15}$ Para identificar os municípios com eliminação da transmissão alcançada e aqueles em processo de eliminação da transmissão, foi apurado o número de casos autóctones registrados no período de 2013 a 2016. 0 índice parasitário anual (IPA), classificado como baixo (0,1 a 9,9 casos por 1.000 hab.), médio $(10,0$ a 49,9/1.000 hab.) e alto (maior ou igual a $50,0 / 1.000$ hab.), ${ }^{16}$ foi utilizado para identificação do risco de contrair a doença.

Desenvolveu-se um algoritmo automatizado para calcular os limites inferior (LIC) e superior (LSC) do diagrama de controle e para apurar o número de casos autóctones, conforme reuniões realizadas com a equipe do PNCM em 17 de janeiro de 2017. Seus resultados, juntamente com o IPA, permitiram classificar os municípios entre os seguintes grupos:

Grupo 1A - Eliminação alcançada - municípios sem registros de casos autóctones durante quatro anos consecutivos (2013 a 2016).

Grupo $2 \mathrm{~A}$ - Em processo de eliminação - municípios sem registro de casos autóctones durante três anos consecutivos (2014 a 2016).

Grupo 2B - Em processo de eliminação - municípios sem registro de casos autóctones durante dois anos consecutivos (2015 a 2016).

Grupo 2C - Em processo de eliminação - municípios que registraram casos autóctones no período de 2013 a 2016, porém com IPA $\leq 1$ caso/1.000 hab. em 2016.

Grupo 3A - Em busca da redução - municípios com IPA>1 caso/1.000 hab., com variação no número de casos abaixo do LIC, e que em nenhuma semana ultrapassaram o LSC, em 2016.

Grupo 3B - Em busca da redução, com incidência estável - municípios com IPA > 1 caso/1.000 hab. e com variação no número de casos entre o LIC e o LSC, durante 52 semanas consecutivas ou mais, em 2016.

Grupo 3C - Em busca da redução, com epidemia municípios com IPA $>1$ caso/1.000 hab. e com número de casos acima do LSC, em qualquer semana de 2016.

A duração da redução da transmissão da malária foi arbitrada em três categorias: (i) curta duração $=1$ a 6 semanas de redução em 2016; (ii) média duração = 7 a 24 semanas de redução; (iii) longa duração = 25 ou mais semanas de redução.

A definição de epidemia utilizada foi a mesma de Medronho e Perez ${ }^{17}$ e da OMS, ${ }^{18}$ ou seja, 'Ocorrência de casos acima do esperado'. Toda frequência semanal, superior a um caso, que ultrapassou o LSC foi considerada uma epidemia. A duração da epidemia, igualmente, foi arbitrada em três categorias: (i) curta duração $=1$ a 6 semanas epidêmicas; (ii) média duração $=7$ a 24 semanas; (iii) longa duração $=25$ semanas ou mais.

0 padrão de distribuição espacial das epidemias nos municípios pautou-se nos índices global e local de Moran. ${ }^{19}$ A dependência espacial das epidemias, representada estatisticamente pela autocorrelação espacial, foi analisada testando-se a variável 'proporção de semanas epidêmicas' em locais distintos do espaço (polígonos municipais), comparando-se 0 valor encontrado em cada município com o valor da mesma variável nos municípios vizinhos, nos anos de 2015 e 2016, por meio dos índices global e local de Moran.

0 Moran Map permitiu a visualização das áreas homogêneas ao distribuir os municípios da região em quadrantes - Q1 +/+, Q2 -/-, Q3 +/- e Q4 -/+ conforme método utilizado por Braz et al. ${ }^{2} 0$ índice global de Moran estimou quanto o valor da variável 'proporção de semanas epidêmicas' em um município foi dependente dos valores dessa mesma variável no conjunto de municípios da região. Valores positivos, entre $0 \mathrm{e}+1$, indicaram autocorrelação direta; $\mathrm{e}$ valores negativos, entre 0 e - 1 , autocorrelação inversa. 0 índice local de Moran identificou os clusters de municípios epidêmicos. A hipótese nula testada foi de independência espacial em cada município, com significância de 5\%. 
Para verificação dos municípios com autocorrelação positiva direta, persistente, utilizaram-se as notificações de malária de 2015 e 2016.

Foi construído o que se denominou 'indicador de prioridades', composto por outros seis indicadores: (i) número de casos novos de malária no ano; (ii) IPA; (iii) porte populacional; (iv) proporção de casos de malária por Plasmodium falciparum em relação ao total de casos da doença; (v) proporção de semanas epidêmicas; e (vi) ocorrência de epidemias. Este indicador de prioridades foi utilizado para classificar os municípios em seis estratos hierárquicos considerados prioritários: Estrato 1-municípios cujo número de casos foi considerado alto ( $>332$ casos por ano, conforme medida separatriz do $3^{\circ}$ quartil) e com IPA também alto ( $\geq 50$ casos/1.000 hab.).

Estrato 2 - municípios cujo número de casos foi considerado alto e com grande porte populacional ( $\geq 100$ mil hab.).

Estrato 3 - municípios cujo número de casos foi considerado alto e com proporção de Plasmodium falciparum $\geq 10 \%$.

Estrato 4 - municípios cujo número de casos foi considerado alto e com autocorrelação positiva direta da proporção de semanas epidêmicas (quadrante do Moran Map =1).

Estrato 5 - municípios cujo número de casos foi considerado alto e com registro de epidemias.

Estrato 6 - demais municípios cujo número de casos foi considerado alto.

Os arquivos eletrônicos, com dados dos casos de malária, foram obtidos do Sivep-Malária em 31 de janeiro de 2017, mediante autorização formal do PNCM/Ministério da Saúde. Todas as análises deste estudo consideraram os casos autóctones de malária segundo o município provável de infecção.

Os tamanhos das populações dos municípios foram extraídos do sítio eletrônico do Departamento de Informática do Sistema Único de Saúde (Datasus). ${ }^{20}$ As malhas dos mapas foram obtidas do Laboratório de Geoprocessamento do Instituto de Comunicação e Informação Cientifica e Tecnológica em Saúde (ICICT), da Fundação Instituto Oswaldo Cruz (Fiocruz).

Para tratamento e análise dos dados, foram usados os programas eletrônicos Epi Info 3.5.4 (https://wwwn. cdc.gov/epiinfo/html/prevVersion.htm) e TerraView 4.2.2 (http://www.dpi.inpe.br/terraview/php/dow. php?body=DowFiles).
O estudo foi aprovado pela Coordenação-Geral de Pós-Graduação/Vice-Presidência de Ensino, Informação e Comunicação da Fiocruz em 17/12/2014: matrícula $\mathrm{n}^{\circ}$ 82957-812014.

Por utilizar apenas dados secundários agregados, o projeto atendeu às considerações éticas previstas na Resolução do Conselho Nacional de Saúde (CNS) $n^{0} 466$, de 12 de dezembro de 2012, estando isento de avaliação por Comitê de Ética em Pesquisa.

\section{Resultados}

A distribuição dos 808 municípios da Amazônia brasileira, conforme o nível de variação da incidência da malária em 2016, identificou: 337 (41,7\%) municípios no Grupo 1A; 54 no Grupo 2A; 58 no Grupo 2B; 286 (35,4\%) no Grupo 2C; 15 no Grupo 3A; nenhum no Grupo 3B; e 58 no Grupo 3C. A maioria dos municípios do Grupo 1A estava localizada no Tocantins (38,0\% do total do grupo), Maranhão (32,3\%) e Mato Grosso $(24,6 \%)$. Os municípios do Grupo 1A representaram $50,2 \%$ do total de municípios no Maranhão, 58,9\% em Mato Grosso e 92,1\% no Tocantins. Em situação oposta, a maioria dos municípios epidêmicos (Grupo 3C) distribuiu-se entre Amazonas (37,9\%), Roraima (15,5\%), Amapá (13,8\%), Pará (13,8\%) e Acre (10,3\% do total do grupo). A estratificação do Grupo 3C identificou epidemias nos municípios de Roraima (60,0\% do total de seus municípios), Amapá (50,0\%), Amazonas (35,5\%), Acre (27,3\%), Rondônia (7,7\%), Pará (5,6\%) e Mato Grosso (0,7\%) (Figura 1).

A análise da estrutura espacial das epidemias de malária mostrou que 0 índice global de Moran encontrado para a variável 'proporção de semanas epidêmicas' foi de 0,4 em 2015 e 0,5 em 2016 ( $p=0,01)$.

Em 2015, detectaram-se 57 municípios com autocorrelação positiva direta $(Q 1+/+), 235$ municípios com autocorrelação positiva inversa (Q2 -/-) e 15 municípios com autocorrelação negativa (Q4 -/+). Não houve municípios com autocorrelação negativa no quadrante $3(\mathrm{Q} 3+/-)$. Detectaram-se 498 municípios com autocorrelação estatisticamente não significativa $(\mathrm{p}>0,05)$. Os 57 municípios do Q1 pertenciam aos estados do Acre $(n=7)$, Amazonas $(n=29)$, Amapá $(n=8)$, Pará $(n=7)$ e Roraima $(\mathrm{n}=6)$ (Figura $2-2015)$.

Em 2016, o número de municípios no Q1 foi reduzido para 46 (19,3\%), em relação ao ano anterior, e 


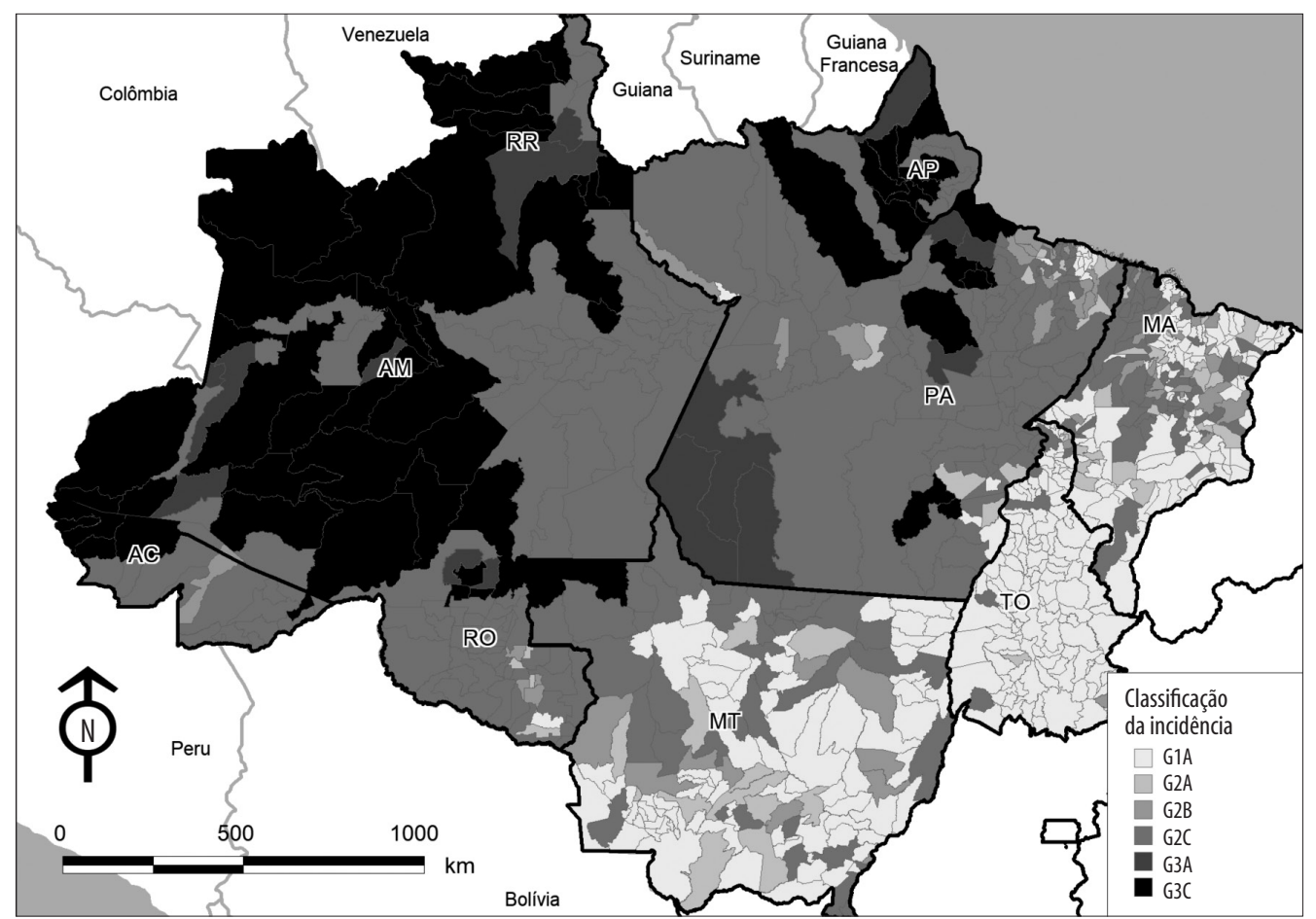

Legenda:

G1A = eliminação alcançada

$G 2 A=$ em processo de eliminação, sem transmissão durante três anos.

$\mathrm{G} 2 \mathrm{~B}=$ em processo de eliminação, sem transmissão durante dois anos.

$\mathrm{G} 2 \mathrm{C}=$ em processo de eliminação, com IPA $\leq 1$ caso/1.000 hab.

$\mathrm{G} 3 \mathrm{~A}=$ em busca da redução, com redução.

$\mathrm{G} 3 \mathrm{C}=$ em busca da reducão, com epidemia.

Nota: nenhum município foi classificado no grupo G3B - em busca da redução; com incidência estável.

\section{Figura 1 - Distribuição dos municípios segundo a classificação da variação da incidência da malária, Amazônia brasileira, 2016}

outros 225 foram classificados no Q2. Somente dois municípios foram encontrados no Q3 e 18 no Q4. Os municípios do Q1 localizaram-se no Acre $(\mathrm{n}=8)$, Amazonas $(n=13)$, Amapá $(n=10)$, Pará $(n=10)$ e Roraima ( $\mathrm{n}=5)$ (Figura $2-2016)$. No mesmo ano, constaram-se 514 municípios com autocorrelação estatisticamente não significativa $(\mathrm{p}>0,05)$.

Verificou-se que 32 municípios persistiram com autocorrelação positiva direta $(Q 1+/+)$ em 2015 e 2016, distribuídos no Acre $(\mathrm{n}=5)$, Amazonas $(\mathrm{n}=9)$, Amapá $(n=8)$, Pará $(n=7)$ e Roraima $(n=3)$. Em 2015, a quantidade de semanas epidêmicas nesses 32 municípios variou de 3 em Ferreira Gomes (AP) a 52 em Pedra Branca do Amaparí (AP); a média, por município, foi de 21 semanas epidêmicas, com desvio-padrão (DP) = 14. Em 2016, nos 32 municípios, o número de semanas epidêmicas variou de 3 em Atalaia do Norte (AM) a 51 em São Gabriel da Cachoeira (AM); a média foi de 20 semanas epidêmicas $(\mathrm{DP}=13)$.
Os 32 municípios epidêmicos com autocorrelação positiva direta persistente nos dois anos avaliados formaram quatro agrupamentos distintos. Do primeiro agrupamento (Figura 3-A1), composto por sete municípios situados no Acre $(\mathrm{n}=5)$ e Amazonas $(\mathrm{n}=2)$, cinco fazem fronteira com o Peru: Cruzeiro do Sul, Mâncio Lima e Marechal Thaumaturgo, no Acre; e Guajará e Atalaia do Norte, no Amazonas. Do segundo agrupamento (Figura 3-A2), composto por dez municípios situados no Amazonas $(\mathrm{n}=7)$ e Roraima $(n=3)$, dois fazem fronteira com a Colômbia - Japurá e São Gabriel da Cachoeira, no Amazonas - e três com a Venezuela - Barcelos e Santa Isabel do Rio Negro, no Amazonas; e Iracema, em Roraima. Do terceiro agrupamento (Figura 3-A3), composto por nove municípios situados no Pará (1) e Amapá (8), o município de Almeirim (PA) faz fronteira com o Suriname, e Laranjal do Jari (AP) com a Guiana Francesa. E do quarto agrupamento (Figura 3-A4), composto por 


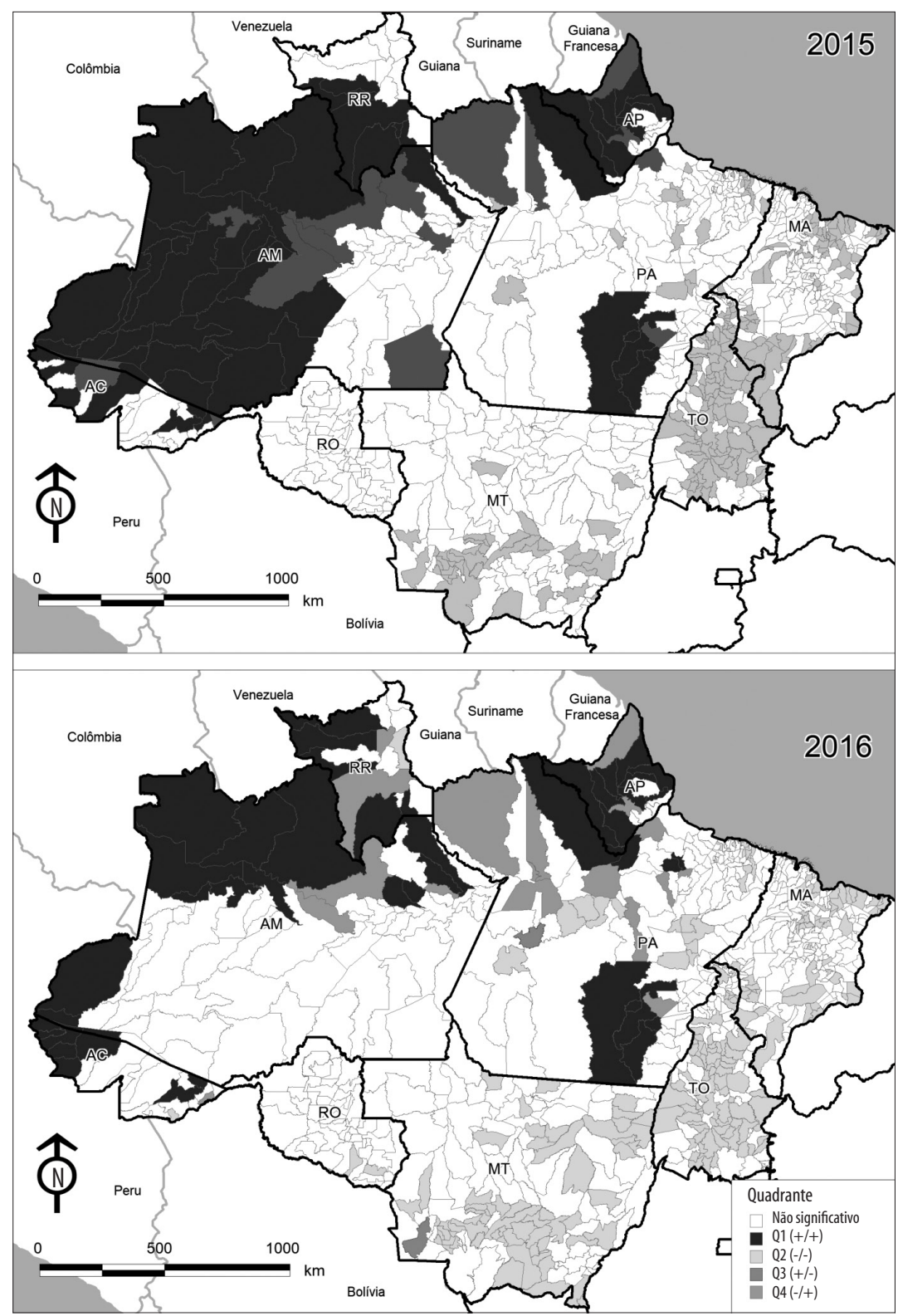

Legenda:
$Q 1(+/+)=$ autocorrelação positiva direta.
$Q 2(-/)=$ autocorrelação positiva inversa.
$Q 3(+/-)=$ autocorrelação negativa.
$04(-/+)=$ autocorreção negativa.

Figura 2 - Moran Map para o indicador 'proporção de semanas epidêmicas' nos municípios da área endêmica, Amazônia brasileira, 2015 e 2016

seis municípios, todos pertencentes ao estado do Pará, nenhum se situa em área de fronteira.

0 indicador de prioridades identificou 71 municípios entre os estratos 2 e 6, em 2016. Neste ano, não houve nenhum município no estrato 1 . 0 s 71 municípios registraram 136.182 casos da doença, cor- respondendo a 94,7\% do total da Amazônia brasileira. A população desses municípios representou 19,3\% do total da região. 0 IPA médio encontrado foi de 25,2 casos/1.000 hab.; e a proporção de $P$. falciparum, de $9,9 \%$ para o conjunto dos munícipios (Figura 4 e Tabela 1). 


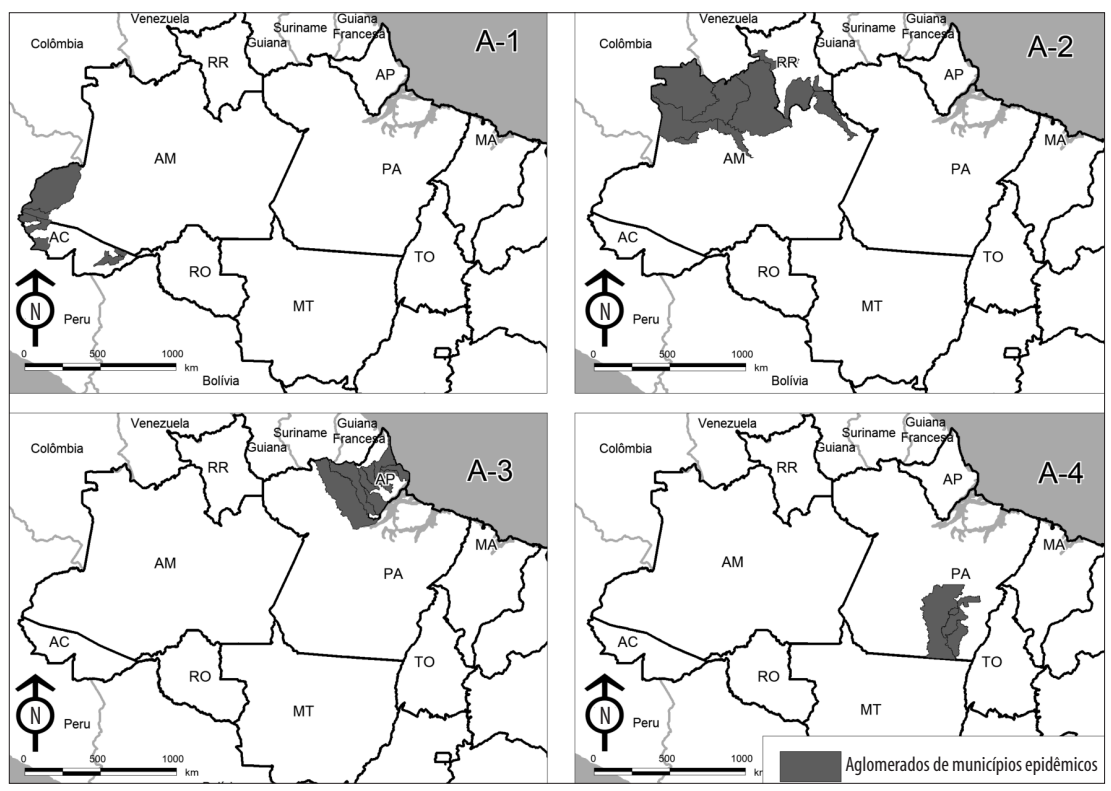

Legenda:

A-1 = Agrupamento 1

A-2 $=$ Agrupamento 2

A-3 $=$ Agrupamento 3

A-4 = Agrupamento 4

Figura 3 - Agrupamentos de municípios epidêmicos com autocorrelação positiva direta - Q1 (+/+) - persistente durante dois anos, Amazônia brasileira, 2015 e 2016

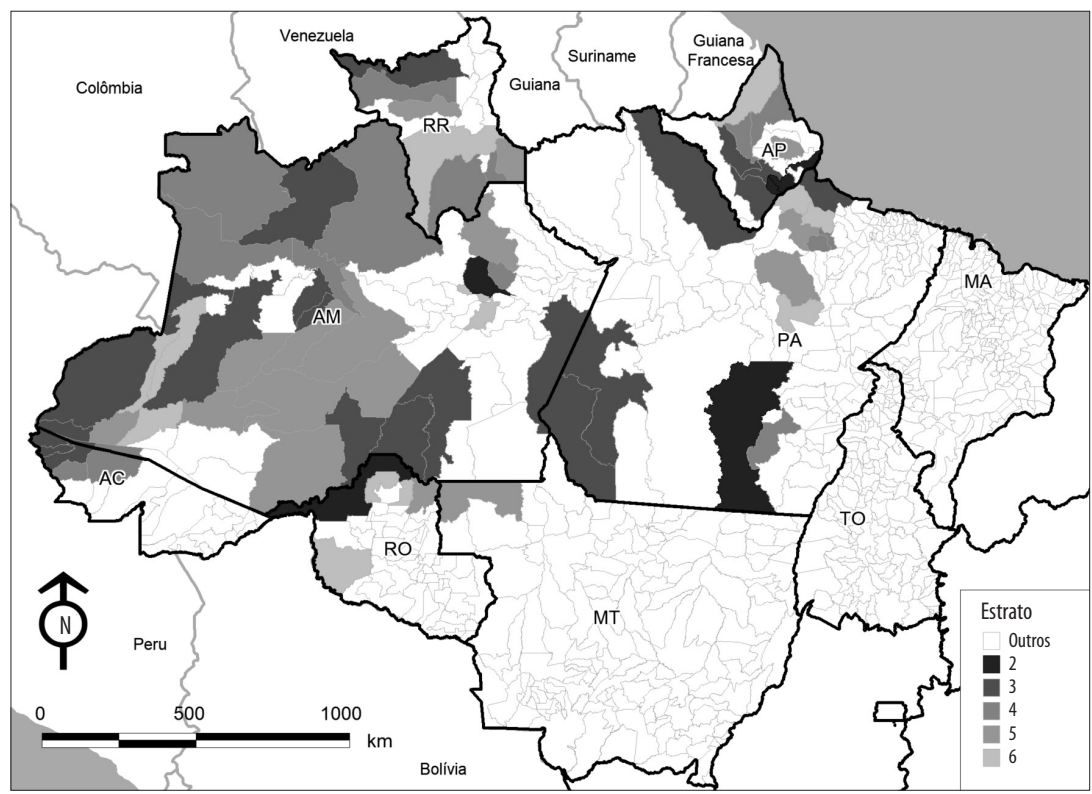

Legenda:

Estrato 2 = municípios com mais de 332 casos por ano e população $\geq 100$ mil hab.

Estrato 3 = municipios com mais de 332 casos por ano e P. falciparum $\geq 10 \%$.

Estrato $4=$ municípios com mais de 332 casos por ano e com quadrante do Moran Map $=1$.

Estrato $5=$ municípios com mais de 332 casos por ano e com registro de epidemias.

Estrato $6=$ demais municípios com mais de 332 casos por ano.

Outros = municípios com menos de 332 casos por ano.

Figura 4 - Municípios classificados entre os estratos 2 e $6(n=71)$ do indicador de prioridades, e respectivos indicadores utilizados para o cálculo das prioridades no controle da malária, Amazônia brasileira, 2016 
Tabela 1 - Municípios classificados, segundo o indicador de prioridades, entre 2 e 6 ( $n=71)$, e respectivos indicadores utilizados para o cálculo das prioridades no controle da malária, Amazônia brasileira, 2016

\begin{tabular}{|c|c|c|c|c|c|c|c|c|c|c|}
\hline Código & Município & UF $^{\mathrm{a}}$ & População & $\begin{array}{l}\text { Porte } \\
\text { populacional }\end{array}$ & $\begin{array}{l}\text { Lâminas } \\
\text { positivas }\end{array}$ & $I^{P A}$ & $\begin{array}{l}\text { \% de casos por } \\
\text { P. falciparum }\end{array}$ & $\begin{array}{l}\text { Número de } \\
\text { semanas } \\
\text { epidêmicas }\end{array}$ & $\begin{array}{l}\text { Quadrante } \\
\text { Moran Map }\end{array}$ & $\begin{array}{c}\text { Estrato } \\
\text { prioritário }\end{array}$ \\
\hline 130260 & Manaus & $A M$ & 2.094.391 & Grande & 10.338 & 0,5 & - & 12 & 1 & 2 \\
\hline 110020 & Porto Velho & Ro & 511.219 & Grande & 3.400 & 0,7 & 15,9 & - & 0 & 2 \\
\hline 160060 & Santana & AP & 113.854 & Grande & 1.656 & 1,5 & 1,4 & 14 & 0 & 2 \\
\hline 160030 & Macapá & AP & 465.495 & Grande & 1.297 & 0,3 & 3,1 & 4 & 0 & 2 \\
\hline 150730 & São Félix do Xingu & PA & 120.580 & Grande & 403 & 0,3 & 1,2 & 23 & 1 & 2 \\
\hline 120020 & Cruzeiro do Sul & $A C$ & 82.075 & Médio & 20.969 & 25,5 & 17,7 & 23 & 1 & 3 \\
\hline 120033 & Mâncio Lima & $A C$ & 17.545 & Pequeno & 8.471 & 48,3 & 13,5 & 43 & 1 & 3 \\
\hline 120042 & Rodrigues Alves & $A C$ & 17.464 & Pequeno & 6.827 & 39,1 & 22,3 & 47 & 1 & 3 \\
\hline 130360 & Santa Isabel do Rio Negro & $A M$ & 23.092 & Médio & 2.895 & 12,5 & 23,4 & 43 & 1 & 3 \\
\hline 130020 & Atalaia do Norte & $A M$ & 18.599 & Pequeno & 2.534 & 13,6 & 19,2 & 3 & 1 & 3 \\
\hline 160040 & Mazagão & AP & 19.981 & Pequeno & 2.520 & 12,6 & 28,4 & 21 & 1 & 3 \\
\hline 130165 & Guajará & $A M$ & 16.085 & Pequeno & 2.280 & 14,2 & 19,3 & 21 & 1 & 3 \\
\hline 150360 & Itaituba & PA & 98.485 & Médio & 2.235 & 2,3 & 20,3 & 0 & 0 & 3 \\
\hline 160015 & Pedra Branca do Amapari & AP & 14.560 & Pequeno & 2.023 & 13,9 & 10,9 & 29 & 1 & 3 \\
\hline 130170 & Humaitá & $A M$ & 52.354 & Médio & 1.422 & 2,7 & 22,3 & 19 & 0 & 3 \\
\hline 140002 & Amajari & RR & 11.285 & Pequeno & 1.128 & 10,0 & 27,2 & 11 & 1 & 3 \\
\hline 160053 & Porto Grande & AP & 20.143 & Médio & 843 & 4,2 & 20,3 & 2 & 4 & 3 \\
\hline 150050 & Almeirim & PA & 33.282 & Médio & 807 & 2,4 & 30,0 & 27 & 1 & 3 \\
\hline 130230 & Jutaí & $A M$ & 16.200 & Pequeno & 730 & 4,5 & 19,0 & 5 & 0 & 3 \\
\hline 130090 & Canutama & $A M$ & 15.312 & Pequeno & 661 & 4,3 & 31,2 & 13 & 0 & 3 \\
\hline 130406 & Tabatinga & AM & 62.346 & Médio & 644 & 1,0 & 12,4 & - & 0 & 3 \\
\hline 130002 & Alvarães & $A M$ & 15.729 & Pequeno & 571 & 3,6 & 16,8 & - & 0 & 3 \\
\hline 150250 & Chaves & PA & 22.821 & Médio & 531 & 2,3 & 16,4 & 4 & 0 & 3 \\
\hline 130290 & Maués & $A M$ & 61.110 & Médio & 500 & 0,8 & 21,6 & 9 & 0 & 3 \\
\hline 130370 & Santo Antônio do Içá & AM & 23.378 & Médio & 494 & 2,1 & 30,8 & 3 & 0 & 3 \\
\hline 130426 & Uarini & $A M$ & 13.276 & Pequeno & 456 & 3,4 & 16,7 & 4 & 0 & 3 \\
\hline 150375 & Jacareacanga & PA & 41.487 & Médio & 441 & 1,1 & 10,9 & - & 0 & 3 \\
\hline 130270 & Manicoré & $A M$ & 53.890 & Médio & 429 & 0,8 & 14,9 & 1 & 0 & 3 \\
\hline 130380 & São Gabriel da Cachoeira & AM & 43.831 & Médio & 13.130 & 30,0 & 0,1 & 51 & 1 & 4 \\
\hline 130040 & Barcelos & $A M$ & 27.589 & Médio & 3.946 & 14,3 & 8,2 & 18 & 1 & 4 \\
\hline 150770 & São Sebastião da Boa Vista & PA & 25.540 & Médio & 2.002 & 7,8 & - & 17 & 1 & 4 \\
\hline 160020 & Calçoene & AP & 10.345 & Pequeno & 1.816 & 17,6 & 0,9 & 18 & 1 & 4 \\
\hline 150280 & Curralinho & PA & 32.881 & Médio & 1.371 & 4,2 & 0,3 & 12 & 1 & 4 \\
\hline 140005 & Alto Alegre & RR & 16.053 & Pequeno & 1.225 & 7,6 & 9,6 & 20 & 1 & 4 \\
\hline 140047 & Rorainópolis & RR & 27.756 & Médio & 1.139 & 4,1 & 1,2 & 9 & 1 & 4 \\
\hline 150543 & Ourilândia do Norte & PA & 31.359 & Médio & 1.079 & 3,4 & 0,4 & 45 & 1 & 4 \\
\hline 160005 & Serra do Navio & AP & 5.025 & Pequeno & 1.040 & 20,7 & 3,6 & 21 & 1 & 4 \\
\hline 120060 & Tarauacá & $A C$ & 39.427 & Médio & 954 & 2,4 & 7,5 & 4 & 1 & 4 \\
\hline 130210 & Japurá & $A M$ & 4.660 & Pequeno & 882 & 18,9 & 4,5 & 27 & 1 & 4 \\
\hline 130356 & Rio Preto da Eva & $A M$ & 31.274 & Médio & 721 & 2,3 & - & 6 & 1 & 4 \\
\hline 140050 & São João da Baliza & RR & 7.629 & Pequeno & 578 & 7,6 & - & 10 & 1 & 4 \\
\hline 130280 & Maraã & $A M$ & 18.477 & Pequeno & 490 & 2,7 & 0,4 & 15 & 1 & 4 \\
\hline 120039 & Porto Walter & $\mathrm{AC}$ & 11.059 & Pequeno & 378 & 3,4 & 8,7 & 19 & 1 & 4 \\
\hline 150180 & Breves & PA & 99.080 & Médio & 2.792 & 2,8 & 0,7 & 23 & 0 & 5 \\
\hline 130353 & Presidente Figueiredo & $A M$ & 33.703 & Médio & 2.028 & 6,0 & 0,3 & 47 & 0 & 5 \\
\hline
\end{tabular}


Tabela 1 - Municípios classificados, segundo o indicador de prioridades, entre 2 e 6 (n=71), e respectivos indicadores utilizados para o cálculo das prioridades no controle da malária, Amazônia brasileira, 2016

\begin{tabular}{|c|c|c|c|c|c|c|c|c|c|c|}
\hline Código & Município & UFa $^{a}$ & População & $\begin{array}{l}\text { Porte } \\
\text { populacional }\end{array}$ & $\begin{array}{l}\text { Lâminas } \\
\text { positivas }\end{array}$ & $\mathrm{IPA}^{\mathrm{b}}$ & $\begin{array}{c}\% \text { de casos por } \\
\text { P. falciparum }\end{array}$ & $\begin{array}{l}\text { Número de } \\
\text { semanas } \\
\text { epidêmicas }\end{array}$ & $\begin{array}{l}\text { Quadrante } \\
\text { Moran Map }\end{array}$ & $\begin{array}{c}\text { Estrato } \\
\text { prioritário }\end{array}$ \\
\hline 130240 & Lábrea & AM & 44.071 & Médio & 1.981 & 4,5 & 2,4 & 1 & 0 & 5 \\
\hline 110013 & Machadinho D'0este & RO & 37.899 & Médio & 1.711 & 4,5 & 0,6 & 7 & 0 & 5 \\
\hline 130120 & Coari & AM & 83.929 & Médio & 1.646 & 2,0 & 0,2 & 2 & 0 & 5 \\
\hline 130180 & Ipixuna & $\mathrm{AM}$ & 27.587 & Médio & 1.565 & 5,7 & 8,2 & 7 & 0 & 5 \\
\hline 150580 & Portel & PA & 59.322 & Médio & 1.304 & 2,2 & 0,1 & 16 & 0 & 5 \\
\hline 130420 & Tefé & $\mathrm{AM}$ & 62.230 & Médio & 1.114 & 1,8 & 9,2 & 1 & 0 & 5 \\
\hline 160070 & Tartarugalzinho & AP & 15.665 & Pequeno & 922 & 5,9 & 0,9 & 29 & 0 & 5 \\
\hline 140030 & Mucajaí & RR & 16.618 & Pequeno & 712 & 4,3 & 4,6 & 20 & 0 & 5 \\
\hline 510325 & Colniza & MT & 34.885 & Médio & 669 & 1,9 & 0,3 & 2 & 0 & 5 \\
\hline 130195 & Itamarati & $\mathrm{AM}$ & 8.153 & Pequeno & 638 & 7,8 & 5,3 & 3 & 0 & 5 \\
\hline 130410 & Tapauá & $A M$ & 18.039 & Pequeno & 572 & 3,2 & 1,0 & 1 & 0 & 5 \\
\hline 130100 & Carauari & $\mathrm{AM}$ & 28.111 & Médio & 552 & 2,0 & 1,1 & 5 & 0 & 5 \\
\hline 140023 & Caroebe & RR & 9.331 & Pequeno & 372 & 4,0 & 0,3 & 33 & 0 & 5 \\
\hline 150070 & Anajás & PA & 28.012 & Médio & 2.635 & 9,4 & 8,1 & - & 0 & 6 \\
\hline 110080 & Candeias do Jamari & RO & 24.719 & Médio & 1.253 & 5,1 & 2,2 & - & 0 & 6 \\
\hline 160050 & Oiapoque & AP & 24.892 & Médio & 922 & 3,7 & 3,4 & - & 4 & 6 \\
\hline 150030 & Afuá & PA & 37.778 & Médio & 621 & 1,6 & 3,9 & - & 4 & 6 \\
\hline 150548 & Pacajá & PA & 45.596 & Médio & 614 & 1,3 & 0,7 & - & 0 & 6 \\
\hline 130140 & Eirunepé & $\mathrm{AM}$ & 34.461 & Médio & 605 & 1,8 & 1,7 & - & 0 & 6 \\
\hline 130390 & São Paulo de Olivença & $\mathrm{AM}$ & 37.300 & Médio & 455 & 1,2 & 2,4 & - & 0 & 6 \\
\hline 130185 & Iranduba & $\mathrm{AM}$ & 46.703 & Médio & 414 & 0,9 & 0,0 & - & 0 & 6 \\
\hline 110094 & Cujubim & RO & 21.720 & Médio & 406 & 1,9 & - & - & 0 & 6 \\
\hline 140020 & Caracaraí & RR & 20.537 & Médio & 396 & 1,9 & 0,5 & - & 4 & 6 \\
\hline 110010 & Guajará-Mirim & RO & 47.048 & Médio & 358 & 0,8 & - & 2 & 0 & 6 \\
\hline 130060 & Benjamin Constant & AM & 40.417 & Médio & 336 & 0,8 & 6,3 & 4 & 0 & 6 \\
\hline 130110 & Careiro & $\mathrm{AM}$ & 36.922 & Médio & 333 & 0,9 & 0,9 & 1 & 0 & 6 \\
\hline Total & - & - & 5.413 .671 & - & 136.182 & 25,2 & 9,9 & - & - & - \\
\hline
\end{tabular}

a) UF:Unidades da Federaçãa.

b) IPA: índice parasitário anual.

\section{Discussão}

Este estudo mostrou que mais de $40,0 \%$ dos municípios da Amazônia brasileira já alcançaram a eliminação da transmissão da malária e estão concentrados nos estados do Maranhão, Mato Grosso e Tocantins. Quase metade dos municípios encontrava-se em processo de eliminação da transmissão. Naqueles que ainda apresentavam transmissão, 32 formavam agrupamentos epidêmicos persistentes em 2015 e 2016, exigindo abordagens diferenciadas no controle da infecção. Nesse sentido, o indicador de prioridades agrupou os municípios hierarquicamente, para melhor direcionar as ações de controle e para subsidiar o monitoramento simultâneo da variação da incidência de malária nos níveis municipal, estadual e federal, com vistas à eliminação da transmissão da doença.

Nos municípios epidêmicos, a variabilidade na duração das epidemias (entre 1 e 51 semanas) pode estar relacionada a fatores como o desmatamento de áreas extensas, grandes migrações com formação de aglomerações desprotegidas, e a falta de estrutura da Saúde, conforme fatores identificados em dois estudos: um deles sobre a influência de alterações ambientais na distribuição da malária na Amazônia brasileira; ${ }^{21}$ e 0 outro, sobre a perspectiva de eliminação da transmissão da doença no país. ${ }^{22}$ Os 58 municípios epidêmicos detectados no grupo 3C deste estudo estão próximos dos 50 observados no monitoramento da malária na Amazônia brasileira, realizado pelo Ministério da Saúde em 2015. ${ }^{23}$ 
Os municípios com epidemias de longa duração foram mais frequentes nos estados com maior incidência da malária, como Acre, Amazonas, Roraima e Amapá. Essas epidemias podem estar relacionadas às limitações operacionais dos serviços de saúde, insuficiência financeira e de recursos humanos e, em algumas localidades, ferramentas inadequadas para interromper a transmissão, conforme observação do grupo de especialistas que apresentou proposta de agenda para erradicação da malária em nível global. ${ }^{24}$ Pesquisa sobre tendências na incidência da malária na Amazônia brasileira, no período de 2004 a 2013, também identificou que os quatro estados mencionados neste parágrafo estão entre aqueles que representam maiores riscos de se contrair malária na região, apesar de Roraima apresentar tendência de redução no período. ${ }^{25}$

0 padrão espacial da distribuição das epidemias nos municípios formou agrupamentos conforme a duração do fenômeno epidêmico. 0 índice global de Moran constatou que a variável 'proporção de semanas epidêmicas' estava correlacionada positivamente no espaço, confirmando a dependência espacial das epidemias entre o conjunto de municípios da região. Isto sinaliza que a transmissão da malária, em determinado município, pode estar relacionada, ecologicamente, a outros municípios vizinhos, mostrando a necessidade de uma ação integrada de controle da malária entre eles. Os resultados encontrados no presente estudo corroboram os achados de uma pesquisa sobre dependência espacial das epidemias de malária, que apontou autocorrelação em $2003(0,4), 2007(0,6)$ e $2010(0,5){ }^{2}$

Nenhum município foi detectado no grupo com incidência estável, provavelmente devido ao ponto de corte utilizado para classificação (IPA>1 caso/1.000 hab.). Estudo para monitoramento da malária na Amazônia brasileira revelou que, geralmente, os municípios com incidência estável ou esperada são aqueles com baixa incidência da doença, situação na qual é possível planejar a eliminação da transmissão, ${ }^{26}$ exigindo empenho redobrado das áreas de vigilância e controle.

A situação atual da vigilância e controle da malária demanda a análise de diversos indicadores, conforme mostrado aqui. Entre esses indicadores, já consagrados pelo PNCM, estão o índice parasitário anual (IPA), a proporção de Plasmodium falciparum e o número absoluto de casos. ${ }^{23} \mathrm{~A}$ autocorrelação espacial das epidemias é outro indicador recomendado para moni- toramento da doença. ${ }^{2}$ Outrossim, deve-se considerar o porte populacional dos municípios, dada a possibilidade de agravamento da transmissão em áreas mais povoadas e dotadas de condições ambientais favoráveis à proliferação do vetor.

A análise estratificada desses indicadores ofereceu subsídios importantes para as ações de vigilância e controle da endemia. Entretanto, o exame conjunto dos indicadores - pelo indicador de prioridades - apontou para a relevância da definição de áreas prioritárias no sentido de evitar a pulverização de recursos humanos, financeiros e logísticos, ao identificar que apenas 8,3\% dos municípios da região concentravam 95\% dos casos de malária. 0 uso de um indicador composto é incomum no controle da malária; porém, ele tem sido muito importante para a vigilância em saúde, por exemplo, no Programa Nacional de Imunizações (PNI) ${ }^{27}$ Assim, 0 indicador de prioridades se apresenta como uma alternativa possível também para subsidiar o PNCM, podendo se readequar caso seja necessário.

Algumas limitações deste trabalho, como a não incorporação das notificações de casos dos municípios situados fora da Amazônia brasileira, devem ser observadas. Essas informações serão consideradas, principalmente para a declaração dos municípios que alcançaram a eliminação da transmissão da malária. Outros indicadores importantes, relacionados ao controle de vetores, não foram incluídos por não fazerem parte do escopo da análise.

Após diversos planos emergenciais, a incidência da malária na Amazônia brasileira encontra-se em processo de efetivo declínio, conforme apontam estudos sobre a situação epidemiológica da doença na região. ${ }^{28}$ As dificuldades para redução da transmissão são maiores nos locais com baixa transmissão, exigindo maior organização e esforço da vigilância e controle. Este é o grande desafio que ora se impõe, com vistas aos objetivos de desenvolvimento sustentável das Nações Unidas, especialmente 0 que prevê acabar com as epidemias de malária e outras doenças até $2030{ }^{29}$ Nesse sentido, a condição político-organizacional poderá ser 0 fator diferencial para o alcance da eliminação sustentável da transmissão da malária nos municípios com indicação epidemiológica. A prioridade política poderá ser buscada com a inserção dos indicadores malariométricos nos planos anuais e plurianuais de saúde, por estados e municípios, garantindo os recursos orçamentários e financeiros necessários para as ações sustentáveis de vigilância e controle da doença. 
Qualquer descuido em relação ao controle da malária poderá causar retrocessos nos resultados alcançados, conforme já ocorreu nos planos anteriores de contenção da doença. Os dados de 2017 apresentam incremento de $51 \%$ no número de casos de malária notificados na Amazônia brasileira, em relação a 2016, ${ }^{30}$ reforçando a necessidade de novas intervenções e intensificação nas ações de vigilância e controle da doença. Novos estudos são necessários para verificar se o incremento da infecção ocorreu nos municípios com transmissão persistente ou naqueles onde a transmissão da doença foi eliminada.

O cenário atual do controle da malária na Amazônia brasileira é promissor, embora requeira a utilização

\section{Referências}

1. World Health Organization. World malaria report 2016 [Internet]. Geneva: World Health Organization; 2016 [cited 2018 Apr 6]. 148 p. Available in: http:// www.who.int/malaria/publications/world-malariareport-2016/report/en/

2. Braz RM, Guimarães RF, Carvalho Júnior OA, Tauil PL. Spatial dependence of malaria epidemics in municipalities of the Brazilian Amazon. Rev Bras Epidemiol. 2014 jul-set;17(3):615-28.

3. Silveira AC, Rezende DF. Avaliação da estratégia global de controle integrado da malária no Brasil. Brasília: Organização Pan-Americana da Saúde; 2001.

4. Deane LM. Os grandes marcos na história do controle da malária. Rev Soc Bras Med Trop. 1992 outdez;25(supl 2):12-22.

5. Ministério da Saúde (BR). Secretaria de Vigilância em Saúde. Programa nacional de controle da Malária [Internet]. Brasília: Ministério da Saúde; 2003 [citado 2018 abr 13]. 132 p. Disponível em: http:// bvsms.saude.gov.br/bvs/publicacoes/programa_nac_ prev_malaria.pdf

6. Loiola CCP, Silva CJM, Tauil PL. Controle da malária no Brasil: 1965 a 2001. Rev Panam Salud Publ. 2002 abr;11(4):235-44.

7. Tauil PL. Avaliação de uma nova estratégia de controle da malária na Amazônia brasileira [tese]. Brasília: Universidade de Brasília; 2002.

8. The Global Fund. Portfolio of grants malaria [Internet]. Geneva: The Global Fund; 2010 [cited 2010 mar 20]. 32 p. Available in: http://www. theglobal-fund.org/programs/portfolio/?countryID=B RA\&lang=en de novas abordagens direcionadas à eliminação da transmissão nos municípios onde ela persiste. Os indicadores propostos neste estudo poderão ampliar as opções analíticas disponíveis para vigilância e controle da malária na região da Amazônia brasileira.

\section{Contribuição dos autores}

Braz RM e Barcellos C contribuíram com a concepção e delineamento do estudo, análise e interpretação dos resultados, redação e revisão crítica relevante de seu conteúdo intelectual, aprovaram a versão final do manuscrito e são responsáveis por todos os aspectos do trabalho, garantindo sua precisão e integridade.

9. Ladislau JLB, Leal MC, Tauil PL. Avaliação do plano de intensificação das ações de controle da malária na região da Amazônia legal, Brasil, no contexto da descentralização. Epidemiol Serv Saude. 2006 abrjun;15(2):9-20.

10. Ministério da Saúde (BR). Secretaria de Vigilância em Saúde. Plano de eliminação da Malária no Brasil (Preliminar) [Internet]. Brasília: Ministério da Saúde; 2016 [citado 2017 abr 15]. 38 p. Disponível em: http://portalarquivos.saude.gov.br/images/pdf/2017/ janeiro/04/Plano-eliminacao-malaria-pub.pdf

11. Ministério da Saúde (BR). Secretaria de Vigilância em Saúde. Sivep-malária. Sistema de informações de vigilância epidemiológica da malária [Internet]. Brasilia: Computorized Systems; 2003 [citado 2018 abr 13]. Disponível em: www.saude.gov.br/sivep_malaria

12. Werneck GL, Struchiner CJ. Estudos de agregados de doença no espaço-tempo: conceitos, técnicas e desafios. Cad Saúde Pública. 1997 outdez;13(4):611-24.

13. Barcellos CC, Sabroza PC, Peiter P, Rojas LI. Organização espacial, saúde e qualidade de vida: análise espacial e uso de indicadores na avaliação de situações de saúde. Inf Epidemiol SUS. 2002 julset;11(3):129-38.

14. Brasil. Casa Civil. Lei $n^{0} 5.173$, de 27 de outubro de 1966. Dispõe sobre o plano de valorização econômica; extingue a Superintendência do Plano de Valorização Econômica da Amazônia (SPVEA); cria a Superintendência de Desenvolvimento da Amazônia (SUDAM), e dá outras providências. Diário Oficial da República Federativa do Brasil, Brasília, DF, 1966 out 31; Seção 1. 
15. Braz RM, Duarte EC, Tauil PL. Epidemiology of malaria in the municipality of Cruzeiro do Sul, State of Acre, Brazil, in 2010: uses of a control chart at the local level. Rev Soc Bras Med Trop. 2012 julago;45(4):526-9.

16. Rede Interagencial de Informações para Saúde. Indicadores de dados básicos: conceitos e aplicações [Internet]. Brasília: Rede Interagencial de Informações para Saúde; 2008 [citado 2018 abr 6]. 350 p. Disponível em: http://www.ripsa.org. br/2014/10/30/indicadores-basicos-para-a-saude-nobrasil-conceitos-e-aplicacoes-livro-2a-edicao-2008-2/

17. Medronho RA, Perez MA. Distribuição das doenças no espaço e no tempo. In: Medronho RA, Carvalho DM, Bloch KV, Luiz RR, Werneck GL. Epidemiologia. São Paulo: Atheneu; 2003.

18. World Health Organization. Disease outbreaks [Internet]. Geneva; 2017 [cited 2017 Nov 8]. Available in: http://www.who.int/topics/disease_ outbreaks/en/

19. Câmara C, Carvalho MS, Cruz OG, Correa V. Análise espacial de áreas. In: Empresa brasileira de Pesquisa Agropecuária - Embrapa. Análise espacial de dados geográficos. Druck S, Carvalho MS, Câmara G, Monteiro AMV, Orgs. Planaltina (DF): Embrapa; 2004. p. 157-206.

20. Ministério da Saúde (BR). Departamento de Informática do SUS. População residente no Brasil, segundo Município. 1996 a 2010 [Internet]. 2010 [citado 2010 fev 2]. Disponível em: http://tabnet. datasus.gov.br/cgi/tabcgi.exe?ibge/cnv/popbr.def

21. Vasconcelos CH, Novo EMLM, Donalisio MR. Uso do sensoriamento remoto para estudar a influência de alterações ambientais na distribuição da malária na Amazônia brasileira. Cad Saúde Pública. 2006 mar;22(3):517-26.

22. Tauil PL. The prospect of eliminating malaria transmission in some regions of Brazil. Mem Inst Oswaldo Cruz. 2011 Jan-Aug;106(Suppl 1):105-6.

23. Ministério da Saúde (BR). Secretaria de Vigilância em Saúde. Malária: monitoramento dos casos no Brasil em 2014. Bol Epidemiol [Internet]. 2015 [citado 2017 set 10];46(25): 1-5. Disponível em: http://portalarquivos2.saude.gov.br/images/pdf/2015/ agosto/18/2015-009---Mal--ria-para-publica----0.pdf
24. Alonso PL, Brown G, Arevalo-Herrera M, Binka F, Chitnis C, Collins F, et al. A research agenda to underpin malaria eradication. PLoS Medicine. 2011 Jan;8(1):1-5.

25. Lima ISF, Lapouble OMM, Duarte EC. Time trends and changes in the distribution of malaria cases in the Brazilian Amazon Region, 2004-2013. Mem Inst Oswaldo Cruz. 2017 Jan;112(1):8-18.

26. Braz RM, Duarte EC, Tauil PL. Algoritmo para monitoramento da incidência da malária na Amazônia brasileira, 2003 a 2010. Rev Panam Salud Publica. 2014 jan;35(3):186-92.

27. Braz RM, Domingues CMAS, Teixeira AMS, Luna EJA. Classification of transmission risk of vaccinepreventable diseases based on vaccination indicators in Brazilian municipalities. Epidemiol Serv Saúde. 2016 Oct-Dec;25(4):745-54.

28. Lapouble OMM, Santelli ACFS, Muniz-Junqueira MI. Situação epidemiológica da malária na região amazônica brasileira, 2003 a 2012. Rev Panam Salud Publica. 2015 jul;38(4):300-6.

29. Organização das Nações Unidas. Centro de Informação das Nações Unidas para o Brasil. Transformando nosso mundo: a agenda 2030 para o desenvolvimento sustentável [Internet]. Brasília: Centro de Informação das Nações Unidas para o Brasil; 2015 [citado 2018 abr 6]. 49 p. Disponível em: https://nacoesunidas.org/wp-content/ uploads/2015/10/agenda2030-pt-br.pdf

30. Ministério da Saúde (BR). Secretaria de Vigilância em Saúde. Número de casos de malária notificados e diferença percentual entre 2016 e 2018 [Internet]. Brasília: Ministério da Saúde; 2018 [citado 2018 abr 18]. https://public.tableau.com/profile/mal. ria.brasil\#!/vizhome/MiniSivep1518_2018_04_10/ casos_notificados_2018_regio_Amaznica 


\begin{abstract}
Objective: to identify areas where malaria transmission has been eliminated and levels of malaria incidence variation in the Brazilian Amazon in 2016, and to present an indicator of priorities for control actions. Methods: an ecological study was conducted with data from the Malaria Epidemiological Surveillance Information System (SivepMalaria); municipalities were classified into three groups - elimination achieved, in the process of elimination and in search of reduction -; a composite indicator was created to stratify municipalities prioritized for control actions. Results: a total of 337 (41.7\%) municipalities were found to have achieved elimination, 398 (49.3\%) were in the process of elimination and 73 (9.0\%) were in search of reduction; the priority indicator created identified 71 municipalities that accounted for 95\% of cases. Conclusion: the vast majority of municipalities have already achieved elimination of malaria transmission or are in the process of eliminating transmission; the priority indicator may contribute to targeting malaria control actions.
\end{abstract}

Keywords: Malaria; Monitoring; Spatial Analysis; Ecological Studies.

\section{Resumen}

Objetivo: identificar áreas con eliminación de transmisión de malaria y la variación de la incidencia de esta enfermedad en la Amazonía brasileña en 2016 y presentar indicadores de prioridades de acción de control. Métodos: se realizó un estudio ecológico con datos del Sistema de Información de Vigilancia Epidemiológica de Malaria (Sivep-Malaria); los municipios fueron clasificados en tres grupos-eliminación alcanzada; proceso de eliminación; y busca de reducción-; se creó un indicador para estratificar los municipios prioritarios para acciones de control. Resultados: se detectaron 337 (41,7\%) municipios con eliminación alcanzada, 398 (49,3\%) en proceso de eliminacióny 73 (9,0\%) en busca de reducción; el indicador de prioridades creado identificó 71 municipios que concentraron 95\% de casos. Conclusión: la gran mayoría de los municipios ya alcanzaron la eliminación de la transmisión de malaria o están en proceso; el indicador de prioridades puede contribuir para la dirección de acciones de control de la infección.

Palabras-clave: Malaria; Monitoreo; Análisis Espacial; Estudios Ecológicos.

Recebido em 10/01/2018

Aprovado em 10/04/2018 